\title{
Association between Clq gene polymorphisms and autoimmune thyroid diseases
}

Qiuming Yao', Jie Li², Xiaofei An', Wenjuan Jiang', Qiu Qin', Ronghua Song', Ni Yan', Danfeng Li', Yanfei Jiang', Wen Wang', Liangfeng Shi', Jin-an Zhang'

\begin{abstract}
Objective: In the present study, we aimed to assess the associations of C1q gene polymorphisms with autoimmune thyroid diseases (AITD) susceptibility. Subjects and methods: A set of 1,003 AITD patients (661 with Graves' disease and 342 with Hashimoto's thyroiditis) and 880 ethnicallyand geographically-matched controls from Chinese Han population were included. Five common single nucleotide polymorphisms (SNPs) (rs294185, rs292001, rs682658, rs665691 and rs294179) in C1q gene locus were genotyped. Frequencies of genotypes and alleles were compared between patients and controls, and haplotype analysis was also performed. Results: There was no statistically significant difference between AITD patients and controls in the frequencies of alleles of rs294185 $(P=0.41), r s 292001(P=0.71), r s 682658(P=0.68), r s 665691(P=0.68)$ and rs294179 $(P=0.69)$. There was also no statistically significant difference between AITD patients and controls in the frequencies of genotypes of rs294185 ( $P=0.72)$, rs292001 ( $P=0.89)$, rs682658 $(P=0.83)$, rs665691 $(P=0.90)$ and rs294179 ( $P=0.43)$. Stratified analyses showed that none of those five SNPs in C1q gene were associated with Graves' disease or Hashimoto's thyroiditis (all P values $>0.05$ ). Haplotype analysis revealed that there were no obvious genetic associations of $\mathrm{C1q}$ gene polymorphisms with AITD susceptibility. Conclusions: We, for the first time, identified the associations between C1q gene SNPs and AITD, and our findings suggested that five common SNPs in C1q gene were not associated with AITD susceptibility in Chinese Han population. Arch Endocrinol Metab. 2017;61(3):337-42.
\end{abstract}

Keywords

Autoimmune thyroid diseases; C1q; single nucleotide polymorphism
Department of Endocrinology, Jinshan Hospital of Fudan University, Shanghai, China ${ }^{2}$ Department of Nephrology, Xi'an Central Hospital, Xi'an, China

Correspondence to: Jin-an Zhang Department of Endocrinology, Jinshan Hospital of Fudan University, 1508 Longhang Road 201508 - Shanghai, People's Republic of China zhangjinan@hotmail.com

Received on May/26/2016 Accepted on Oct/18/2016 DOI: 10.1590/2359-3997000000256

\section{INTRODUCTION}

A utoimmune thyroid diseases (AITD) are common autoimmune disorders in endocrinologic system, affecting about $5 \%$ of overall population (1). AITD is characterized by immune imbalance and autoantibodies towards thyroid. As the most common type of AITD, Hashimoto's thyroiditis (HT) mainly causes hypothyroidism, and it is characterized by lymphocytic infiltration and presence of thyroid peroxidase antibodies (TPOAb) or thyroglobulin antibody $(\mathrm{TgAb})$. Graves' disease (GD) is another main type of AITD, which is characterized by hyperthyroidism due to overproduction of thyroid hormones induced by specific auto-antibodies against thyrotropin receptor (TSHR).

It has been known that many factors are involved in the initiation and development of AITD, such as genetic factors, environmental factors and nutritional elements, such as iodine intake and infection $(2,3)$. However, the pathogenesis of AITD remains unclear. In the past decade, several genetic polymorphisms have been found to be associated with AITD susceptibility, such as genetic polymorphisms in the genes encoding TSHR, human leukocyte antigen $(H L A)$ and cytotoxic T lymphocyteassociated antigen-4 (CTLA4) (4-7). Other genetic polymorphisms associated with AITD susceptibility have also been reported, such as polymorphisms in the CD40, IL-17, FCRL3 and protein tyrosine phosphatase-22 (PTPN22) genes (8-11). However, the above-mentioned genetic polymorphisms can only explain part of gene susceptibility to AITD, and other genetic polymorphisms are believed to have important roles in AITD, which need to be explored in future studies.

Complement is a vital part of innate immune system in human body, which can be activated by three different pathways (12). The classical pathway 
of complement activation is characterized by the binding of $\mathrm{Clq}$ to immune complexes (13). Clq is a recognition component in the classical pathway, and it can help solubilize immune complexes and aid in the clearance of apoptotic debris (14). The gene coding region for $\mathrm{Clq}$ is localized on chromosome lp34-36 and consists of three genes, ClqA,ClqB and $C l q C$ (15). Several single nucleotide polymorphisms (SNPs) have been found in the Clq gene, such as rs292001, rs682658, rs665691 and rs294179. SNPs in the $\mathrm{Cl} q$ gene have been reported to be associated with several common autoimmune diseases, such as systemic lupus erythematosus (SLE) and rheumatoid arthritis. However, the roles of Clq gene SNPs in AITD susceptibility remain poorly explored. In the present study, the associations of five common SNPs in $\mathrm{Cl} q$ gene with AITD susceptibility were examined in order to identify additional risk variants for AITD susceptibility. We, for the first time, clarified the roles of $C l q$ gene SNPs in AITD susceptibility in Chinese Han population.

\section{SUBJECTS AND METHODS}

\section{Subjects}

The present study was a case-control study, and all subjects were from Chinese Han population. A set of 1,003 AITD patients (661 with GD and 342 with HT) and 880 ethnically- and geographicallymatched controls were included. All controls were healthy and unrelated to AITD patients. The AITD patients were recruited in the outpatient department of Jinshan Hospital of Fudan University (Shanghai, China). AITD patients were diagnosed according to clinical guidelines, which were described in details in our previously published studies (16). The diagnosis of GD was based on the presence of clinical and laboratory biochemical hyperthyroidism with diffuse goiter, decreased TSH value as well as increased levels of free thyroid hormones and anti-thyroid stimulating hormone receptor antibody (TRAb) (+). HT was diagnosed by the presence of an enlarged thyroid and TPOAb $(+)$ or TgAb $(+)$. The healthy subjects without thyroid goiters, autoimmune diseases and family history were randomly recruited from health examination center of Jinshan Hospital of Fudan University (Shanghai, China) and used as controls. All the controls presented no $\operatorname{TRAb}(+), \operatorname{TPOAb}(+)$ or $\operatorname{TgAb}(+)$. The research was approved by the Ethics
Committee of Jinshan Hospital of Fudan University (Shanghai, China), and informed consents were obtained from all included participants.

\section{Genotyping}

About $2 \mathrm{~mL}$ peripheral venous blood was collected from patients and controls. Genomic DNA was extracted from the collected blood samples, and the concentration and purity of DNA samples were determined by Nano Drop 2000 Spectro-photometer (Thermo, USA). From Hapmap CHB database, five SNPs of Clq gene (rs292001/rs682658/rs294185/ rs66569l/rs294179) were selected according to the predisigned eligibility criteria as follows: ( 1 ) the frequency of minor allele was greater than 0.10 ; and (2) P value for Hardy-Weinberg equilibrium (HWE) was greater than 0.001 . The genotyping of five SNPs of $C l q$ was conducted by using ligase detection reaction (LDR) platform (16). The target DNA sequences of those five SNPs of Clq gene were amplified using multiplex polymerase chain reaction (PCR) method. The sequences of primers for those five SNPs were as follows:

(1) rs294185:

Forward: 5'-ACCCCAGCTTTGACATTTGC-3'; Reverse: 5'-GGTGTGGTCTCAGTTTTAGG-3'; (2) rs292001:

Forward: 5'-TCCTAGTCCAAAGCAGACCA-3'; Reverse: 5'-GTTCAGGTACCACATGTAGG-3'; (3) rs66569l:

Forward: 5'-AAGCATTCTCAGGGTCCAAG-3'; Reverse: 5'-CCTTAACTGATGGGATGCTC-3'; (4) rs294179:

Forward: 5'-GCACATCTTGCCTTTGTCTG-3'; Reverse: 5'-CCTGTGCTGAACTTCAGGAG-3'; (5) rs682658:

Forward: 5'-ACTTGGCCCTAGGAGTCCCT-3'; Reverse: 5'-CAGCCCCATAATGCAGTATC-3'.

\section{Statistical analysis}

Statistical analyses were carried out by using SPSS (version 17.0). Chi-square test was used to detect the difference in the frequencies of genotypes and alleles between patients and controls. The association between SNPs and AITD susceptibility was firstly assessed, and then stratified analyses were performed based on the types of AITD. Haplotype analysis was also conducted using Haploview 4.0 platform. P value less than 0.05 was considered statistically significant. 


\section{RESULTS}

\section{Demographic and clinical characteristics of subjects}

Table 1 shows the demographic and clinical characteristics of all the participants in this study. In the GD group, $464(70.2 \%)$ patients were females, $197(29.8 \%)$ patients were males, and the mean age was 36.9 years (Table 1 ). The HT group consisted of 274 (80.1\%) female patients and 68 male patients, and their mean age was 34.8 years (Table 1). In the control group, 587 (66.7\%) individuals were females, $293(33.3 \%)$ individuals were males, and the mean age was 38.8 years (Table 1 ). No significant difference concerning age and gender was observed among those three groups, and all $\mathrm{P}$ values were greater than 0.05 (Table 1 ).

\section{Allele and genotyping results}

Genotype distributions for the loci of rs294185, rs292001, rs665691, rs294179 and rs682658 were all confirmed to HWE in both patients and controls $(\mathrm{P}>0.05)$. Table 2 shows the frequencies of alleles and genotypes for those five SNPs in patients and controls.

No statistically significant difference was observed between AITD patients and controls in the frequencies of alleles of rs294185 $(\mathrm{P}=0.41)$, rs292001 $(\mathrm{P}=0.71)$, rs682658 $(\mathrm{P}=0.68)$, rs665691 $(\mathrm{P}=0.68)$ and $\mathrm{rs} 294179$

Table 1. Demographic and clinical characteristics of all the participants in this study

\begin{tabular}{lccc}
\hline \multicolumn{1}{c}{ Characteristics } & $\begin{array}{c}\text { GD } \\
(\mathbf{N}=\mathbf{6 6 1})\end{array}$ & $\begin{array}{c}\text { HT } \\
(\mathbf{n = 3 4 2})\end{array}$ & $\begin{array}{c}\text { Controls } \\
\mathbf{( N = 8 8 0 )}\end{array}$ \\
\hline Sex (\%) & & & \\
$\quad$ Male & $197(29.8)$ & $68(19.9)$ & $293(33.3)$ \\
$\quad$ Female & $464(70.2)$ & $274(80.1)$ & $587(66.7)$ \\
Age (Mean \pm SD, year) & $36.9 \pm 14.5$ & $34.8 \pm 13.8$ & $38.8 \pm 9.0$ \\
Onset age (Mean \pm SD, year) & $33.9 \pm 14.5$ & $32.4 \pm 13.4$ & $N^{*}$ \\
Thyroid size (\%) & & & \\
$\quad$ Normal & $116(17.6)$ & $48(14.0)$ & $880(100.0)$ \\
$\quad$ First degree & $109(16.5)$ & $56(16.4)$ & $0(0.0)$ \\
$\quad$ Second degree & $347(52.5)$ & $213(62.3)$ & $0(0.0)$ \\
Third degree & $89(13.4)$ & $25(7.3)$ & $0(0.0)$ \\
Family history (\%) & & & \\
$\quad$ Yes & $134(20.27)$ & $70(20.47)$ & $0(0.0)$ \\
No & $527(79.73)$ & $272(79.53)$ & $880(100.0)$ \\
Ophthalmopathy (\%) & & & \\
Yes & $115(17.40)$ & $6(1.75)$ & $0(0.0)$ \\
No & $546(82.60)$ & $336(98.25)$ & $880(100.0)$ \\
\hline
\end{tabular}

*NA: not available.
$(\mathrm{P}=0.69)$ (Table 2$)$. There was also no statistically significant difference between AITD patients and controls in the frequencies of genotypes of rs294185 $(\mathrm{P}=0.72)$, rs292001 ( $\mathrm{P}=0.89)$, rs682658 $(\mathrm{P}=0.83)$, rs66569l $(\mathrm{P}=0.90)$ and rs294179 $(\mathrm{P}=0.43)$ (Table 2).

Stratified analyses showed that none of those five SNPs in Clq gene were associated with GD or HT (all P values $>0.05)($ Table 2).

\section{Haplotype analysis}

Haplotype analysis suggested strong linkage disequilibrium (LD) in those five SNPs of $\mathrm{Cl} q$ gene (Table 3). LD mainly existed between rs665691 and rs292001, rs292001 and rs682658, rs66569l and rs682658, rs294185 and rs294179. Table 4 shows the frequency of each haplotype. However, there were no obvious associations of the haplotypes of block 1 and block 2 with GD or HT (Table 4). Figure 1 shows two detected LD blocks according to D' value, which were rs665691-rs292001-rs682658 and rs294185rs294179, respectively.

\section{DISCUSSION}

Polymorphisms in the complement $\mathrm{Cl} q$ gene have been reported to be associated with several types of autoimmune diseases. However, their roles in AITD still remain unclear. In the present study, we aimed to assess the associations of $\mathrm{Cl} q$ gene polymorphisms with AITD susceptibility. To the best of our knowledge, we, for the first time, clarified the roles of Clq gene SNPs in AITD susceptibility. We recruited a set of 1,003 AITD patients (661 with GD and 342 with HT) and 880 ethnically- and geographically-matched controls from Chinese Han population, and examined the associations of five commonly detected SNPs in $\mathrm{Clq}$ gene with AITD susceptibility. In the present study, we showed that AITD patients and healthy controls had statistically similar frequencies of genotypes and alleles of rs294185, rs292001, rs682658, rs665691 and rs294179 (Table 2). Haplotype analysis, which can provide powerful and conducive analyses in identifying genes associated with complex diseases (17), also did not find obvious associations of $\mathrm{Cl} q$ gene with AITD susceptibility. Therefore, our findings suggested that those five common SNPs in Clq gene (rs294185, rs292001, rs682658, rs66569l and rs294179) were not associated with AITD susceptibility in Chinese Han population. 
Table 2. Genotype distributions and allele frequencies of C1q SNPs in AITD patients and controls

\begin{tabular}{|c|c|c|c|c|c|c|c|c|}
\hline SNPs & $\begin{array}{c}\text { Allele or } \\
\text { genotypes }\end{array}$ & $\begin{array}{c}\text { Controls } \\
\text { (\%) }\end{array}$ & $\begin{array}{l}\text { AITD } \\
(\%)\end{array}$ & $P$ values & $\begin{array}{l}\text { GD } \\
\text { (\%) }\end{array}$ & $P$ values & $\begin{array}{l}\text { HT } \\
(\%)\end{array}$ & $P$ values \\
\hline \multirow{5}{*}{ rs294185 } & $\mathrm{CC}$ & $304(34.5)$ & $361(36.0)$ & \multirow{3}{*}{0.72} & 235 (35.5) & \multirow{3}{*}{0.78} & $126(36.8)$ & \multirow{3}{*}{0.75} \\
\hline & CT & $411(46.7)$ & $466(46.5)$ & & $311(47.0)$ & & $155(45.3)$ & \\
\hline & TT & 165 (18.7) & 176 (17.5) & & 115 (17.4) & & $61(17.8)$ & \\
\hline & $C$ & 1019 (57.9) & 1188 (59.2) & \multirow{2}{*}{0.41} & 781 (59.1) & \multirow{2}{*}{0.51} & 407 (59.5) & \multirow{2}{*}{0.470} \\
\hline & $\mathrm{T}$ & $741(42.1)$ & $818(40.8)$ & & $541(40.9)$ & & 277 (40.5) & \\
\hline \multirow{5}{*}{ rs292001 } & AA & $369(41.9)$ & $410(40.9)$ & \multirow{3}{*}{0.89} & $269(40.7)$ & \multirow{3}{*}{0.88} & $141(41.2)$ & \multirow{3}{*}{0.97} \\
\hline & $A G$ & $398(45.2)$ & 463 (46.2) & & $307(46.4)$ & & $156(45.6)$ & \\
\hline & $\mathrm{GG}$ & $113(12.8)$ & $130(12.9)$ & & 85 (12.9) & & 45 (13.2) & \\
\hline & A & $1136(64.5)$ & $1283(64.0)$ & \multirow{2}{*}{0.71} & 845 (63.9) & \multirow{2}{*}{0.72} & $438(64.0)$ & \multirow{2}{*}{0.81} \\
\hline & G & 624 (35.5) & $723(36.0)$ & & $477(36.1)$ & & $246(36.0)$ & \\
\hline \multirow{5}{*}{ rs665691 } & CC & $368(41.8)$ & $409(40.8)$ & \multirow{3}{*}{0.90} & $269(40.7)$ & \multirow{3}{*}{0.90} & $140(40.9)$ & \multirow{3}{*}{0.94} \\
\hline & $\mathrm{CG}$ & $399(45.3)$ & $463(46.2)$ & & $307(46.4)$ & & $156(45.6)$ & \\
\hline & GG & $113(12.8)$ & $131(13.0)$ & & 85 (12.9) & & 46 (13.5) & \\
\hline & C & 1135 (64.5) & $1281(63.9)$ & \multirow{2}{*}{0.69} & 845 (63.9) & \multirow{2}{*}{0.74} & $436(63.7)$ & \multirow{2}{*}{0.73} \\
\hline & G & 625 (35.5) & $725(36.1)$ & & $477(36.1)$ & & $248(36.3)$ & \\
\hline \multirow{5}{*}{ rs294179 } & AA & 110 (12.5) & 134 (13.4) & \multirow{3}{*}{0.43} & 87 (13.2) & \multirow{3}{*}{0.49} & 47 (13.7) & \multirow{3}{*}{0.66} \\
\hline & $A G$ & $405(46.0)$ & $432(43.1)$ & & $284(43.0)$ & & $148(43.3)$ & \\
\hline & $\mathrm{GG}$ & 365 (41.5) & 437 (43.5) & & $290(43.8)$ & & $147(43.0)$ & \\
\hline & A & 625 (35.5) & 700 (34.9) & \multirow{2}{*}{0.69} & 458 (34.6) & \multirow{2}{*}{0.62} & $242(35.4)$ & \multirow{2}{*}{0.95} \\
\hline & G & 1135 (64.5) & $1306(65.1)$ & & $864(65.4)$ & & 442 (64.6) & \\
\hline \multirow{5}{*}{ rs682658 } & $\mathrm{GG}$ & $116(13.2)$ & $132(13.2)$ & \multirow{3}{*}{0.83} & 86 (13.0) & \multirow{3}{*}{0.81} & 46 (13.5) & \multirow{3}{*}{0.96} \\
\hline & GT & $393(44.7)$ & $461(45.9)$ & & $306(46.3)$ & & $155(45.3)$ & \\
\hline & TT & 371 (42.2) & $410(40.9)$ & & $269(40.7)$ & & $141(41.2)$ & \\
\hline & G & 625 (35.5) & $725(36.1)$ & 069 & 478 (36.2) & 071 & $247(36.1)$ & 078 \\
\hline & $\mathrm{T}$ & 1135 (64.5) & $1281(63.9)$ & 0.09 & 844 (63.8) & 0.11 & 437 (63.9) & 0.10 \\
\hline
\end{tabular}

SNP: single nucleotide polymorphism; AITD: autoimmune thyroid diseases; GD: Graves' disease; HT: Hashimoto's thyroiditis.

Table 3. Linkage disequilibrium in those five SNPs of C1q gene

\begin{tabular}{|c|c|c|c|c|c|}
\hline \multirow{2}{*}{ L1 } & \multirow{2}{*}{ L2 } & \multicolumn{2}{|c|}{ D' } & \multicolumn{2}{|c|}{$\mathbf{r}^{2}$} \\
\hline & & Control & AITD & Control & AITD \\
\hline rs665691 & rs292001 & 1.0 & 0.998 & 0.998 & 0.991 \\
\hline rs292001 & rs682658 & 0.998 & 0.993 & 0.973 & 0.983 \\
\hline rs665691 & rs682658 & 0.988 & 0.991 & 0.975 & 0.983 \\
\hline rs294185 & rs294179 & 0.997 & 0.992 & 0.753 & 0.767 \\
\hline
\end{tabular}

Table 4. Haplotype analysis in AITD patients and controls

\begin{tabular}{lccccccc}
\hline Haplotypes & Control (N, \%) & AITD (N, \%) & P values & GD (N, \%) & P values & HT (N, \%) & P values \\
\hline CAT & $1129(64.2)$ & $1273(63.7)$ & 0.74 & $844(63.8)$ & 0.83 & $429(63.5)$ & 0.73 \\
GGG & $619(35.2)$ & $719(36.0)$ & 0.62 & $477(36.1)$ & 0.62 & $242(35.8)$ & 0.78 \\
CG & $1018(57.8)$ & $1185(59.1)$ & 0.44 & $779(58.9)$ & 0.54 & $405(59.4)$ & 0.49 \\
TA & $624(35.5)$ & $697(34.7)$ & 0.65 & $456(34.5)$ & 0.58 & $241(35.3)$ & 0.94 \\
TG & $117(6.6)$ & $121(6.0)$ & 0.44 & $85(6.4)$ & 0.81 & $36(5.3)$ & 0.21 \\
\hline
\end{tabular}




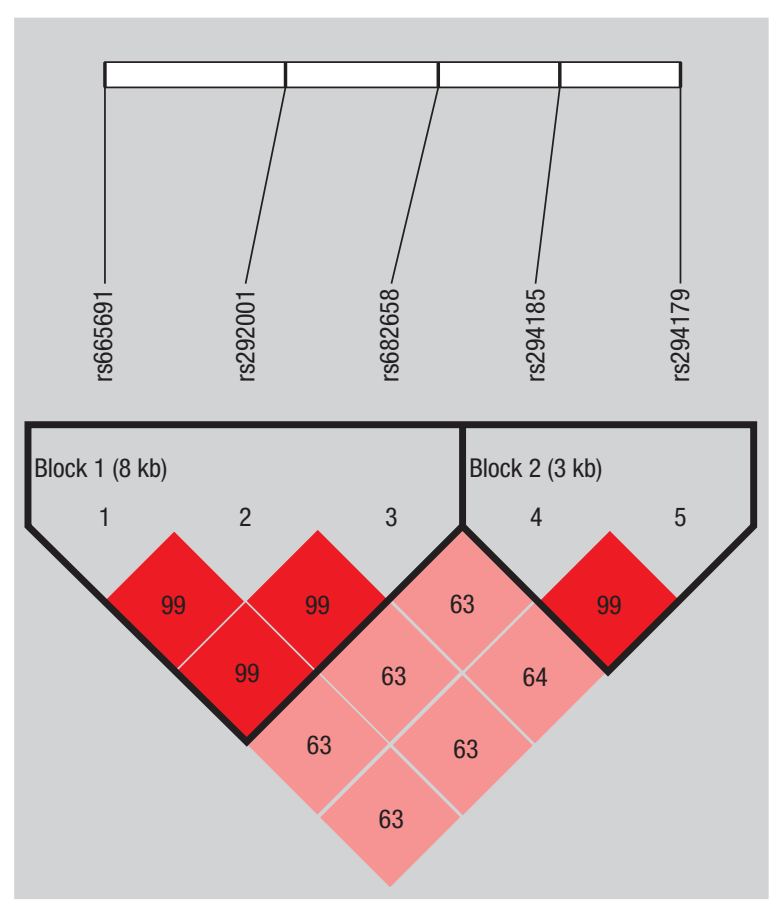

Figure 1. Linkage disequilibrium (LD) block defined by the Haploview 4.2. There were five tag SNPs on the LD map of C1q gene. Patterns of LD between the C1q gene SNPs were shown. The strength of LD measured by D' multiplying 100 was displayed in the red diamonds. A larger number in the cell indicates a higher degree of LD.

The complement system is a vital part of immune system in human body, and it is involved in both innate and adaptive immune systems. As an important part of $\mathrm{Cl}$, Clq plays important roles in the clearance of apoptotic cells and immune complexes (18). Clq deficiency is the first identified single-gene defect, which causes lupus-like disease (19). Patients with Clq deficiency can develop lupus with high penetrance (18). It has been reported that more than $90 \%$ of individuals with complete congenital deficiency of $\mathrm{Clq}$ can develop early-onset photosensitive SLE (14). The presence of anti-Clq has been also strongly correlated with hypocomplementemia, disease activity and renal involvement in SLE patients (20). Several SNPs have been also found in the Clq gene, such as rs292001, rs682658, rs66569l and rs294179. It has been reported that the A allele and AA genotype of Clq rs292001 can be considered a risk factor for juvenile SLE and lupus nephritis in a cohort of Egyptian children (21).

Goulielmos and cols. reported that Clq rs292001 is associated with type 2 diabetes mellitus (22). Other genetic polymorphisms of $\mathrm{Cl} q$ have been also associated with susceptibility to autoimmune diseases, such as rheumatoid arthritis (23). Since $\mathrm{Clq}$ deficiency can result in increased susceptibility to lupus-like autoimmune disease, the genetic polymorphisms of $C l q$ may also have important roles in SLE (24). In addition, genetic deficiencies of $\mathrm{Cl} q$ in mice can also lead to autoimmunity $(25,26)$. The above-mentioned findings suggest that $\mathrm{Clq}$ is intensively involved in autoimmunity, and genetic polymorphisms in $\mathrm{Cl} q$ are possibly associated with some types of autoimmune diseases.

It has been known that many factors are involved in the initiation and development of AITD, such as genetic factors, environmental factors and nutritional factors. However, the exact pathogenesis of AITD still remains poorly explored. Previous studies have suggested that some genetic factors are intensively involved in the initiation of immune responses against the thyroid gland during the development of AITD, such as HLA and CTLA4 (2). AITD is characterized by autoimmunity, and Clq has been suggested to have some roles in AITD. Potlukova and cols. reported that auto-antibodies against $\mathrm{Clq}$ are more prevalent in AITD patients compared with controls (27), while Brohee and cols. reported that circulating immune complexes containing $\mathrm{Clq}$ are also more prevalent in AITD patients than controls (28). All of the above evidence suggests that $\mathrm{Clq}$ gene is probably an important element associated with AITD. However, our data failed to identify obvious associations of those five different SNPs with AITD susceptibility. Therefore, it is necessary to explore the roles of $\mathrm{Cl}$ q gene in AITD in future studies.

There were several limitations in our study. First, the findings from our study were not sufficient to explore the full roles of $\mathrm{Clq}$ gene in AITD, since we only explored five common SNPs in the Clq gene. Future studies can further investigate the associations of other SNPs in the Clq gene with AITD susceptibility. In addition, our study was carried out in only Chinese Han population, which could not be generalized to other ethnical populations. The roles of $\mathrm{Clq}$ gene polymorphisms in AITD susceptibility in Caucasian or African populations need to be studied in future studies. Second, the sample size in our study might not be enough to detect a modest association of $\mathrm{Clq}$ gene with AITD susceptibility. More studies with larger sample size are still required to further identify those SNPs carrying a smaller risk effect. Finally, we did not analyze the gene-environment interaction in our study due to the limitation of study design. Prospective studies in the future may explore the possible gene- 
environment interaction in the associations of $\mathrm{Clq}$ gene polymorphisms with AITD susceptibility. In conclusion, our findings suggested that five common SNPs in Clq gene (rs294185, rs292001, rs682658, rs66569l and rs294179) were all not associated with AITD susceptibility in Chinese Han population. Future studies are required to investigate the associations of those $\mathrm{Clq}$ gene SNPs with AITD susceptibility in Caucasian or African populations. In addition, it is also necessary to explore the associations of other SNPs in $\mathrm{Cl} q$ gene with AITD susceptibility in future studies.

Acknowledgements: authors are grateful to all the participants in this research. The study was financially supported by grants from the National Natural Science Foundation of China (No. 81471004) and the Key Disciplines Development of Shanghai Jinshan District (No. JSZK2015A02).

Disclosure: no potential conflict of interest relevant to this article was reported.

\section{REFERENCES}

1. Tomer $Y$, Huber A. The etiology of autoimmune thyroid disease: a story of genes and environment. J Autoimmun. 2009;32(3-4):231-9.

2. TomerY, Davies TF. Searching for the autoimmune thyroid disease susceptibility genes: from gene mapping to gene function. Endocr Rev. 2003;24:694-717.

3. TomerY, Davies TF. Infection, thyroid disease, and autoimmunity. Endocr Rev. 1993;14:107-20.

4. Tomer Y, Greenberg DA, Concepcion E, Ban Y, Davies TF. Thyroglobulin is a thyroid specific gene for the familial autoimmune thyroid diseases. J Clin Endocrinol Metab. 2002;87:404-7.

5. Liu L, Wu HQ, Wang Q, Zhu YF, Zhang W, Guan LJ, et al. Association between thyroid stimulating hormone receptor gene intron polymorphisms and autoimmune thyroid disease in a Chinese Han population. Endocr J. 2012;59:717-23.

6. Ban Y, Davies TF, Greenberg DA, Concepcion ES, TomerY. The influence of human leucocyte antigen (HLA) genes on autoimmune thyroid disease (AITD): results of studies in HLA-DR3 positive AITD families. Clin Endocrinol (Oxf). 2002;57:81-8.

7. Kavvoura FK, AkamizuT, AwataT, BanY, Chistiakov DA, Frydecka I, et al. Cytotoxic T-lymphocyte associated antigen 4 gene polymorphisms and autoimmune thyroid disease: a meta-analysis. J Clin Endocrinol Metab. 2007;92:3162-70.

8. Heward JM, Brand OJ, Barrett JC, Carr-Smith JD, Franklyn JA, Gough SC. Association of PTPN22 haplotypes with Graves' disease. J Clin Endocrinol Metab. 2007;92:685-90.

9. Ban Y, Tozaki T, Taniyama M, Tomita M, Ban Y. Association of a $\mathrm{C} / \mathrm{T}$ single-nucleotide polymorphism in the $5^{\prime}$ untranslated region of the CD40 gene with Graves' disease in Japanese. Thyroid. 2006;16:443-6.

10. Yan N, Yu YL, Yang J, Qin Q, Zhu YF, Wang X, et al. Association of interleukin-17A and -17F gene single-nucleotide polymorphisms with autoimmune thyroid diseases. Autoimmunity. 2012;45:533-9.
11. Gu LQ, Zhu W, Zhao SX, Zhao L, Zhang MJ, Cui B, et al. Clinical associations of the genetic variants of CTLA-4, Tg, TSHR, PTPN22, PTPN12 and FCRL3 in patients with Graves' disease. Clin Endocrinol (Oxf). 2010;72:248-55.

12. Fujita T, Matsushita M, Endo $Y$. The lectin-complement pathway-its role in innate immunity and evolution. Immunol Rev. 2004;198:185-202.

13. Duncan $A R$, Winter $G$. The binding site for $C 1 q$ on IgG. Nature. 1988;332:738-40.

14. Sontheimer RD, Racila E, Racila DM. C1q, its functions within the innate and adaptive immune responses and its role in lupus autoimmunity. J Invest Dermatol. 2005;125:14-23.

15. Sellar GC, Blake DJ, Reid KB. Characterization and organization of the genes encoding the $\mathrm{A}_{-}, \mathrm{B}-$, and $\mathrm{C}$-chains of human complement subcomponent $\mathrm{C} 1 \mathrm{q}$, the complete derived amino acid sequence of human C1q. Biochem J. 1991;274:481-90.

16. Yan N, Meng S, Zhou J, Xu J, Muhali FS, Jiang W, et al. Association between STAT4 gene polymorphisms and autoimmune thyroid diseases in a Chinese population. Int J Mol Sci. 2014;15:12280-93.

17. Shibata $S$, Saeki H, TsunemiY, KatoT, Nakamura K, KakinumaT, et al. IL-17F single nucleotide polymorphism is not associated with psoriasis vulgaris or atopic dermatitis in the Japanese population. J Dermatol Sci. 2009;53:163-5.

18. Botto M, Walport MJ. C1q, autoimmunity and apoptosis. Immunobiology. 2002;205:395-406.

19. Botto M, Kirschfink M, Macor P, Pickering MC, Wurzner R, Tedesco F. Complement in human diseases: lessons from complement deficiencies. Mol Immunol. 2009;46:2774-83.

20. Flierman R, Daha MR. Pathogenic role of anti-C1q autoantibodies in the development of lupus nephritis-a hypothesis. Mol Immunol. 2007;44:133-8.

21. Mosaad YM, Hammad A, Fawzy Z, El-Refaaey A, Tawhid Z, Hammad EM, et al. C1q rs292001 polymorphism and C1q antibodies in juvenile lupus and their relation to lupus nephritis. Clin Exp Immunol. 2015;182:23-34.

22. Goulielmos GN, Samonis G, Apergi M, Christofaki M, Valachis A, Zervou MI, et al. C1q but not mannose-binding lectin (Mbl2) gene polymorphisms are associated with type 2 diabetes in the genetically homogeneous population of the island of Crete in Greece. Hum Immunol. 2013;74:878-81.

23. Trouw LA, Daha N, Kurreeman FA, Böhringer S, Goulielmos GN, Westra $\mathrm{HJ}$, et al. Genetic variants in the region of the $\mathrm{C} 1 \mathrm{q}$ genes are associated with rheumatoid arthritis. Clin Exp Immunol. 2013;173(1):76-83.

24. Walport MJ. Complement and systemic lupus erythematosus. Arthritis Res. 2002;4:S279-93.

25. 25. Mitchell DA, Pickering MC, Warren J, Fossati-Jimack L, CortesHernandez J, Cook HT, et al. C1q deficiency and autoimmunity: the effects of genetic background on disease expression. J Immunol. 2002;168:2538-43.

26. Warren J, Mastroeni P, Dougan G, Noursadeghi M, Cohen J, Walport MJ, et al. Increased susceptibility of C1q-deficient mice to Salmonella enterica serovarTyphimurium infection. Infect Immun. 2002;70:551-7.

27. Potlukova E, Jiskra J, Limanova Z, Kralikova P, Smutek D, Mareckova $\mathrm{H}$, et al. Autoantibodies against complement $\mathrm{C} 1 \mathrm{q}$ correlate with the thyroid function in patients with autoimmune thyroid disease. Clin Exp Immunol. 2008;153:96-101.

28. Brohee D, Delespesse G, Debisschop MJ, Bonnyns M. Circulating immune complexes in various thyroid diseases. Clin Exp Immunol. 1979;36:379-83. 